\title{
Models for status inconsistency and mobility: a comparison of the approaches by Hope and Sobel with the mainstream square additive model
}

\author{
JOHN HENDRICKX, NAN DIRK DE GRAAF, JAN LAMMERS \& \\ WOUT ULTEE
}

Department of Sociology, University of Nijmegen, P.O. Box 9108, 6500 HK Nijmegen, The Netherlands

\begin{abstract}
This paper is about the analysis of effects of status inconsistency and mobility on a dependent variable. We compare the mainstream square additive baseline model to alternative designs by Hope $(1971,1975)$ and Sobel $(1981,1985)$. Both writers claim that the square additive baseline model also contains some status inconsistency effects. An examination of the relationships between the square additive model, Hope's halfway/difference model, and Sobel's simple diagonal reference model shows that the effects uncovered by Sobel and Hope pertain to the inequality of the effects of the status variables on the dependent variable. These salience difference effects are therefore distinct from the non-additive status inconsistency effects which would be detected using the square additive approach. Less restricted versions of the diagonal reference model, the DM-1 and DM-2 models as well as a recent model by Weakliem (1992), are also examined with regard to additive/non-additive components and symmetry of effects.
\end{abstract}

\section{Introduction}

\author{
I know a discontented gentleman \\ Whose humble means match not his haughty spirit \\ Richard the Third, Act IV. SC. II
}

The purpose of status inconsistency models is to ascertain whether having different ranks on two (or more) status variables affects attitudes and behavior. A wide range of dependent variables have been used, but political radicalism, prejudice/racism, psychological stress and social integration are the more important types. Structural characteristics such as ethnicity, education, occupation, income have typically been used as status variables. In addition, mobility has often been treated as a special case of status inconsistency, using the variables status father/status son. There are substantive differences between status inconsistency and mobility, but also a considerable theoretical overlap. The status variables are transformed to a common scale in most status inconsistency research, in which case the research methods are identical. Henceforth we will use the terms status inconsistency and mobility interchangeably. 
Status inconsistency was a popular research topic in the 1960s. Wilson and Zurcher (1976) list a large number of articles for a wide range of dependent variables. Often cited articles are Lenski $(1954,1956)$, Jackson (1962), Jackson and Burke (1965), Blalock $(1966,1967)$ and Duncan (1966). There was some debate as to the proper model (see Whitt 1983, for an overview), but after this had been resolved in favor of an analysis of variance model, the so-called square additive model, empirical results refuted the predictions.

For many the matter was then closed, but Hope $(1971,1975)$ and Sobel $(1981,1985)$ argued that the square additive model was unsuitable and proposed alternatives. Hope's alternative approach has been applied by Wilson (1979), Zurcher and Wilson (1979), Whitt (1983), and Slomczynski (1989). Sobel's approach has been applied by De Graaf and Ultee (1987, 1990), De Graaf and Ganzeboom (1990), Sorenson (1989), De Graaf (1991), De Graaf and Heath (1992), and Weakliem (1992).

Are there grounds for a resurrection (Wilson 1979) of status inconsistency research? What information can the alternative approaches by Hope and Sobel give that the square additive model cannot, and is this information of interest to researchers? Sobel's approach contains multiplicative terms and requires different estimation techniques: is it totally different from the square additive model and Hope's approach, or does it simply implement the same basic principles in a slightly different fashion?

In order to answer these questions, we will map the relationships between three models for the analysis (using categorical status variables) of status inconsistency and mobility: the mainstream square additive model, Hope's halfway/difference model, and Sobel's diagonal reference ${ }^{1}$ model. Particular attention is given to less restricted versions of the diagonal reference model, the DM-1 and DM-2 models by Sobel, and a recently developed alternative by Weakliem (1992). These derivations have been checked in a re-analysis of the OCG-1 data in Sobel (1981); programs ${ }^{2}$ and results are available on request. When non-equivalent models were being compared, such as the diagonal reference model and the square additive model, the fitted values of the more parsimonious model were used as input for the complex model, in order to check the validity of our derivations.

\section{Background}

Status inconsistency research began as a stratification problem. Lenski (1954) wanted to prove that a uni-dimensional treatment of status would be inappropriate, and posited that if differences between status dimensions could have an effect on a dependent variable, this would prove that these status dimen- 
sions cannot be reduced to an underlying status continuum without important loss of information. Later analyses tended to shift focus to status inconsistency as a determinant of behavior, and theorizing became more socialpsychological in nature.

Jackson (1962) theorized that status inconsistency would affect behavior because it would cause a stressful role conflict, since the status inconsistent ego would wish to ignore his low status dimension, while alter would wish to ignore ego's high status dimension. Geschwender (1967) posited that overor underfulfillment of reward expectations (income, occupation) based on "investments" (race/ethnicity, education) would lead to cognitive dissonance, which could be coped with in three sequential stages: (1) emotional response, (2) social change attempt, (3) social isolation/psychosomatic symptoms. Both authors assumed that effects would be affected by the specific status dimensions in the analysis, and that effects need not be symmetric, e.g. the effect of high education/low occupation need not be the same as the effect of low education/high occupation.

As for research methods, the earliest attempts by Lenski $(1954,1956)$ and Jackson (1962), simply used the status variables to construct a measure of status inconsistency and proceeded with the analysis. The results confirmed their predictions, but others ${ }^{3}$ objected that this could have been due to the status variables themselves, because the effects of these had not been (adequately) controlled.

Blalock (1966) showed that the problem went deeper. If $X_{1}$ and $X_{2}$ are two status variables with continuous scales and $\left(X_{1}-X_{2}\right)$ is treated as the measure of status inconsistency (assuming that the scales have been made comparable, e.g. by standardizing them) then it will be impossible to control for the effects of the status variables and status inconsistency. The equation

$$
Y=b_{1} X_{1}+b_{2} X_{2}+b_{3}\left(X_{1}-X_{2}\right)+\epsilon
$$

will be unidentifiable. $\left(X_{1}-X_{2}\right)$ is a linear transformation of $X_{1}$ and $X_{2}$, making it impossible to derive unique estimates of $b_{1}, b_{2}$ and $b_{3}$.

\section{The square additive model}

The identification problem indicates that in order to determine status inconsistency effects, it is necessary to first account for the effects of the status variables in a baseline model (Duncan 1966). This can be done in an analysis of variance model for status variables with categorical scales, denoting the additive effects as the contribution to explained variance by the status variables, and interaction effects as the contribution by status inconsistency. We 
assume in the following that the status variables have an equal number of categories, which results in a square table of means, for which the diagonal cells are status consistent.

The square additive baseline model for two status variables $X_{1}$ and $X_{2}$, with categories $i, j=1$ to $r$, is specified for respondent $k=1$ to $n$ as:

$$
Y_{i j k}=\hat{\mu}_{i j}+\epsilon_{i j k} \text {, where } \hat{\mu}_{i j}=\mu+\alpha_{i}+\beta_{j} \text {, }
$$

We assume that the constraint $\Sigma_{i=1}^{r} \alpha_{i}=0$ and $\Sigma_{i=1}^{r} \beta_{j}=0$ have been used. ${ }^{4}$ In that case, the square additive model takes the status variables into account by defining expected behavior for each combination of statuses, based on the average behaviors in the categories of the respective status variables.

The full model introduces an interaction term $\gamma_{i j}$ (with $2 r-1$ identification constraints) to test for status inconsistency effects:

$$
\hat{\mu}_{i j}=\mu+\alpha_{i}+\beta_{j}+\gamma_{i j}
$$

If equation (3) explains significantly more variance than equation (2), then there are deviations from the expected behaviors defined by the baseline model. The $\gamma_{i j}$ parameters correspond with "unusual" behaviors associated with a particular combination of statuses and can therefore justifiably be equated with status inconsistency effects. Patterns of these status inconsistency effects could be determined by imposing extra constraints on $\gamma_{i j}$ parameters, e.g. setting certain parameters to equal values or to zero. This would be in accordance with the theoretical expectations of Jackson and Geschwender that only certain types of status inconsistency have effects on a particular dependent variable.

The square additive model became the mainstream model for testing status inconsistency and mobility effects. However, results were largely disappointing. Using a large number of dependent variables, Jackson and Curtis (1972) found significant interaction effects in only $12 \%$ of the hypotheses tested, while those they did find did not replicate across populations. Subsequently, many researchers considered the status inconsistency issue closed.

\section{Hope's approach}

\subsection{The diamond model}

Hope $(1971,1975)$ proposed to rehabilitate status inconsistency. He posited that the square additive model is incorrect, because its main effects also contain some status inconsistency effects. According to his reasoning, the fact that the effects of $\left(X_{1}-X_{2}\right)$, the most logical operationalization of status 
inconsistency, cannot be identified using the square additive model, shows that these effects are already contained in the main effects of $X_{1}{ }^{\prime}$ and $X_{2}$.

In view of Lenski's (1954) original work, Hope felt that it would instead be more appropriate to control for an overall status dimension and to test for the effects of an orthogonal status inconsistency dimension. In 1971 he devised a halfway/difference model for the analysis of variance approach, which he renamed the "diamond model" in 1975, when he also extended his approach to include continuous status variables. We will use the term "diamond model" to refer to analyses with continuous status variables and reserve the term "halfway/difference model" for analysis of variance in which we are primarily interested here.

Hope's (1975) diamond model for continuous variables transforms the status variables into two orthogonal variables for overall status $\left(Z_{1}\right)$ and status inconsistency $\left(Z_{2}\right)$ :

$$
Y=b_{1} Z_{1}+b_{2} Z_{2}+\epsilon
$$

where $Z_{1}=w_{1} X_{1}+w_{2} X_{2}, Z_{2}=w_{3} X_{1}-w_{4} X_{2}$, and $w_{1} w_{3}-w_{2} w_{4}=0$.

However, since the status variables have a common scale, equal weights are a logical choice and Hope's diamond model is usually simplified to:

$$
Y=b_{1}\left(X_{1}+X_{2}\right)+b_{2}\left(X_{1}-X_{2}\right)+\epsilon
$$

By using an overall status dimension as baseline criterion, rather than $X_{1}$ and $X_{2}$ separately, Hope is able to identify the effect of the status inconsistency term $\left(X_{1}-X_{2}\right)$. Equation (5) rotates the original $X$ axes by $135^{\circ}$ so that the line $X_{1}=X_{2}$ becomes the new $Z_{1}$ axis and the line $X_{1}=-X_{2}$ becomes the $Z_{2}$ axis. The line $X_{1}=X_{2}$ can be seen as the ratio of rewards expected for given investments in terms of Geschwender's theory, and the $Z_{2}$ axis indicates how much the actual set of statuses differs from the expected set (cf. also Alschuler 1973).

Hope's diamond model was criticized by House (1978), Sobel (1981) and Zimmerman (1985) because of its use of an overall status dimension. This would correspond with a unidimensional class concept, which many theorists reject in favor of multiple social status dimensions. However, the concept of status inconsistency logically implies the complementary concept of status consistency, which indicates a construct of overall status.

House (1978) also argued that equation (5) will indicate the presence of status inconsistency effects whenever the effects of the status variables on a dependent variable differ: if $\hat{Y}=c_{1} X_{1}+c_{2} X_{2}$, then in equation (5), $b_{1}=$ $\frac{1}{2}\left(c_{1}+c_{2}\right)$ and $b_{2}=\frac{1}{2}\left(c_{1}-c_{2}\right)$. Actually, it is a corollary to the notion of a single, underlying status continuum that the status variables which are manifestations of this latent concept will have effects of approximately the same 
magnitude. This was Lenski's motive for testing for status inconsistency effects in order to falsify the hypothesis of a single, underlying status continuum.

This criticism does clarify what Hope's model in fact does. Equation (5) will explain exactly as much variance of $Y$ as a linear model of $X_{1}$ and $X_{2}$. It only restates the case in terms of the explanatory power of a construct of overall, consistent status and a dimension indicating the disparate strengths of the two status variables in determining the concept measured by the dependent variable. The fact that for example parents' status influences voting behavior more than the respondent's own present status can be of interest, and it might be worthwhile to transform the model so that this information is given. However, effects of this nature are still etfects of the status variables: they have only been restated.

\subsection{The halfway/difference model}

Rotating a square table of means by $135^{\circ}$ results in a diamond shape. However, for categorical status variables, we have chosen Hope's original name, the halfway/difference model. This is specified as:

$$
\hat{\mu}_{i j}=\mu+h_{i}+h_{j}+d_{i}+d_{j}
$$

where $h_{i}=\frac{1}{2}\left(\alpha_{i}+\beta_{i}\right)$ and $d_{i}=\frac{1}{2}\left(\alpha_{i}-\beta_{i}\right)$.

The halfway parameters indicate the average effect on the dependent variable of the row and column variable for each category. This is equivalent to modelling the effects of an underlying, overall status variable. Each difference effect indicates the unequal influence on the dependent variable of the row and column variable for that category: the difference in salience (to use Sobel's term) of the row and column variable.

The halfway/difference model as presented in equation (6) is a transformation of the square additive model, but Hope also proposed submodels by using the polynomial contrast for the difference effects, and testing whether only the linear, or the linear and quadratic terms would fit the data adequately. If a linear constraint is imposed on both the halfway and difference effects, then equation (6) is equivalent to equation (5).

If there are only halfway effects in a halfway/difference model, then the status variables can be treated as a single underlying status variable (for the dependent variable in question). If the difference effects are significant, then the status variables have unequal salience. Hope calls these effects status inconsistency effects, but although they are based on $\left(X_{1}-X_{2}\right)$, we question the appropriateness of the term. Because a halfway/difference model is 
equivalent to the square additive baseline model, status inconsistency effects in the usual sense can still be found by testing for interaction effects. Therefore, at least a distinction must be made between these salience difference "status inconsistency" effects, and status inconsistency effects in the usual, non-additive sense.

Hope's halfway/difference model shows that, just as the (between cells) effects of an ANOVA model can be divided into additive and non-additive components, the additive component in turn can be divided into equal/unequal effect components. It is the "unequal" additive effects component (i.e. difference effects) that contain linear status inconsistency effects and preclude the use of such an effect with the square additive model as baseline: the square additive model already contains the linear status inconsistency effects (and higher order polynomials of the difference effects as well).

The differences in the strengths of the effects of the status variables on behavior will often be of theoretical interest. Especially in the case of mobility research (De Graaf and Ultee 1987, 1990) or the comparison of influence of husbands and wives (Sorenson, 1989; De Graaf and Ganzeboom, 1990), this difference in salience can be of as much interest as non-additive effects. However, these researchers analyzed the difference in salience using Sobel's approach, which is discussed below.

\section{Sobel's approach}

\subsection{The diagonal reference model}

Sobel $(1981,1985)$ criticized both the square additive and Hope's approach. He agreed with Hope that $\left(X_{1}-X_{2}\right)$ is the obvious operationalization of status inconsistency or mobility, and that the fact that such a variable cannot be identified in the square additive model indicates that this model implicitly contains linear status inconsistency effects in its main effects. However, as noted above, he objected to Hope's use of a measure of overall status.

Instead, Sobel proposed to construct a model of the acculturation process that takes place when status inconsistency occurs and to use this as a baseline. The model was originally designed for mobility effects research, but Sobel (1985) proposed that it can be applied to status inconsistency problems as well. Sobel's simple diagonal reference baseline model for a square $r$ by $r$ table of means is:

$$
\hat{\mu}_{i j}=p \mu_{i i}+(1-p) \mu_{j j} \text {. }
$$

The diagonal of the table of means is the focus of interest for Sobel. He 
posited that when acculturation takes place, status inconsistents will take their cues from the status consistents. Status inconsistents will then be faced with two referent behaviors, one for each status value. Consequently, the means of off diagonal cells are a compromise between the mean of the diagonal cell for the score on $X_{1}\left(\mu_{i i}\right)$ and the mean of the diagonal cell for the score on $X_{2}\left(\mu_{j j}\right)$. The parameter $p$ expresses the relative salience of $X_{1}$ and $X_{2}$ If $P>\frac{1}{2}$, then $X_{1}$ has greater salience, since the behavior of status inconsistents resembles the behavior of the status consistents with their score on $X_{1}$ more than the behavior of status consistents with their score on $X_{2}$. Sobel also specified that $p$ lies in the closed interval $[0,1]$. This is not a condition for the model however, it is simply a property of $p$ that it will generally lie between zero and one. The reason for this and the exceptions are discussed below.

Because salience is expressed by a multiplicative parameter, equation (7) is not a linear model. Sobel uses nonlinear least-squares estimation to derive values of $p$ and the values of diagonal cells $\mu_{11}$ to $\mu_{r r}$ of a square table of means, such that these optimally reproduce all values $\mu_{i j}$. This seems to make the diagonal reference model totally different from other models. A first step in facilitating comparison with other models is to incorporate an overall mean, which can be done by rewriting equation (7) as:

$$
\hat{\mu}_{i j}=\mu+p \nu_{i}+(1-p) \nu_{j}, \quad \text { where } \quad \sum_{i=1}^{r} \nu_{i}=0
$$

Table 1 compares the designs of the square additive, halfway, and diagonal reference models for a 3 by 3 table of means. The overall mean is not added to the cells in order to facilitate a comparison of the additive effects; the marginals of the tables contain the additive components.

The relationships between the square additive model and the diagonal reference model can be determined by examining the situation in which both produce the same predicted values. In that case, for the diagonal cells; $\nu_{i}=$ $\alpha_{i}+\beta_{i}=2 h_{i}$. The diagonal values $\mu_{i i}$ in equation (7) can therefore be seen the effects of an underlying continuum, based on equally weighted status variables.

As for the off-diagonal cells, if the models produce the same fitted values, then the additive components will be the same, so

$$
\begin{aligned}
& p \nu_{i}=\alpha_{i} \\
& p\left(\alpha_{i}+\beta_{i}\right)=\alpha_{i} \\
& p=\frac{\alpha_{i}}{\alpha_{i}+\beta_{i}}=\frac{1}{1+\beta_{i} / \alpha_{i}} .
\end{aligned}
$$


Table 1. A comparison of the designs of the square additive model, Hope's halfway model and Sobel's diagonal reference model

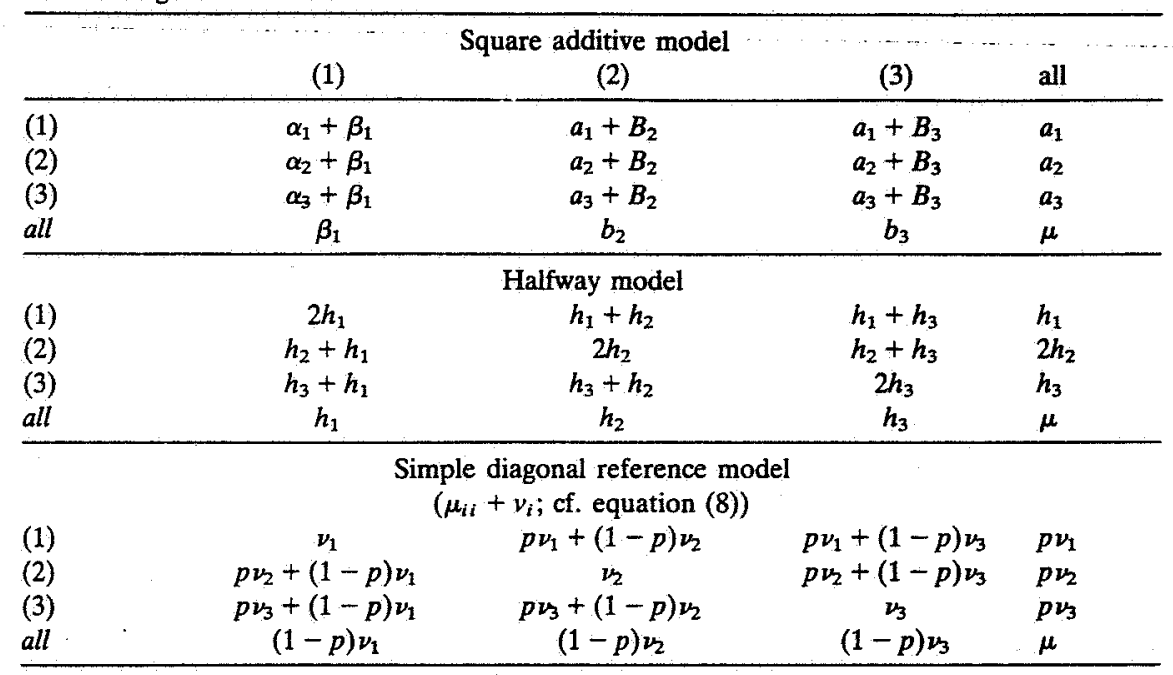

The overall term is not added to cells in order to enhance clarity.

Parameters under the heading "all" are the additive main effect parameters.

Equation (9) clarifies why $p$ usually lies in the interval $[0,1]$. If $\alpha_{i}$ and, $\beta_{i}$ both have the same sign and neither is equal to zero, $\beta_{i} / \alpha_{i}$ will be between zero and infinity, and $p$ will lie between one and zero. If the signs of $\alpha_{i}$ and, $\beta_{i}$ are opposite, then $p$ can have any value. However, in actual analyses, the status variables can be expected to have effects in the same direction (although the effects may both be near zero).

If $p=\frac{1}{2}$, then the main effects are equal, and the halfway model and the diagonal reference model are equivalent (Sobel 1981). $A p \neq \frac{1}{2}$ in Sobel's approach means that the $d_{i}$ values in Hope's model will be unequal to 0 . The distinction is that Sobel expresses unequal salience of the status variables through the ratio of the main effect parameters, while Hope uses the difference between them. ${ }^{5}$

An advantage of Sobel's model is that it is derived from a theory of an acculturation process. However, several criticisms can be raised against this theory. Will status inconsistents really look to the status consistents for cues? How will they know a status inconsistent from a status consistent during superficial initial contacts? What happens when the association between the status variables diminishes? As Sobel notes "But if, for example, the proportion of stayers in a destination class is small, this may decrease the visibility of stayers and diminish their ability to define the sociocultural norms that typify the destination class" (Sobel 1981: 904). 
However, even if these criticisms were to be considered to be strong enough to reject the theory of the acculturation process, the diagonal reference model would still yield valuable insights. An alternative way of looking at the diagonal reference model is in terms of marginal values (cf. Table 1), to be used for the analytical purpose of determining expected cell values. Sobel's model imposes a constant inequality (as opposed to the variable inequality of Hope's difference parameters) on the marginal means: the main effect of $X_{1}$ must be $p /(1-p)$ times the effect of $X_{2}$. The expected behavior tor each situation (each cell) is therefore biased to a constant extent to the average behavior of one of the status variables.

As for the advantages and disadvantages of Sobel's approach versus that of Hope, the simple diagonal reference model expresses unequal salience in a single parameter, whereas the difference variable has $r-1$ (non-redundant) parameters. A linear constraint can be placed on the difference parameters in Hope's model, but will lead to an unacceptable loss of fit unless the main effects of the square additive model are (approximately) linear too. Sobel's model on the other hand, does not even require ordered categories. If the main effects of the status variables have a reasonably similar profile, i.e. are strongly positive and strongly negative for the same categories, then it will be possible to impose the constraint that $\alpha_{i}$ is $p /(1-p)$ times $\beta_{i}$ with no great loss of fit. Sobel's diagonal reference model will explain almost as much variance as the square additive baseline model, with fewer parameters.

This advantage is partially offset by the possibility that $p$ will not be meaningful. If the numerator of the ratio $p$ is based on is zero, then $p$ will be zero irrespective of the denominator; if the denominator is zero, $p$ is undefined. If the effects of $X_{1}$ or the effects of $X_{2}$ on the dependent variable are near zero, then the values of $p$ will become extreme. This will show up in a large value of the asymptotic standard error of $p$. In this situation, the difference parameters of Hope's approach will still be meaningful. However, given the nature of the data used in status inconsistency and mobility analyses, it is not likely that one or both status variables will have near zero effects.

\subsection{The DM-1/DM-2 models}

Sobel felt that in some cases it might be too restrictive to use one salience parameter $p$. For example, if mobility out of a class is strong, then that class might be expected to have less impact than other classes (Sobel 1985: 710). Sobel therefore extended his model to let $p$ vary either with $X_{1}$ (the DM1 model) or with $X_{2}$ (the DM-2 model). His diagonal reference model DM1 is: 


$$
\hat{\mu}_{i j}=p_{i} \mu_{i i}+\left(1-p_{i}\right) \mu_{j j} \text {. }
$$

The DM-2 model is the same as DM- 1 , except that the $X_{2}$ specific weights $p_{j}$ are used. An undesirable property of these models is that they are asymmetrical, in the sense that they require salience of origin versus destination to vary either by origin (DM-1) or destination category (DM-2).

The simple diagonal reference model is nested within the square additive model, and can be supplemented with non-additive status inconsistency effects. The DM-1/DM-2 models, on the other hand, contain a non-additive component. This can be seen by specifying $p_{i}$ in equation (10) as $q+r_{i}$, with $\Sigma_{i-1}^{r} r_{i}=0$. The DM-1 model can then be written as:

$$
\mu_{i j}=q \mu_{i i}+(1-q) \mu_{j j}+r_{i} \mu_{i i}-r_{i} \mu_{j j} \text {. }
$$

All elements in equation (11) are additive, with the exception of the term $-r_{i} \mu_{j j}$. This component must be attributed to unique combinations of $X_{1}$ and $X_{2}$ and should therefore, in the linear modelling scheme, be associated with status inconsistency, rather than incorporated in the baseline model.

However, as rewritten in equation (11), the DM-1 model consists of the simple diagonal reference model plus a status inconsistency term $r_{i}\left(\mu_{i i}-\mu_{j j}\right)$. $\mu_{i i}-\mu_{j i}$ can be seen as a cross pressures component indicating the difference in the behaviors of referents. The parameter $r_{i}$ indicates the relative influence of cross pressures for each origin class.

If the DM-2 model is used, there would be a destination specific parameter $s_{j}$ (with $q+s_{j}=p_{j}$ and $\Sigma_{j=1}^{r} s_{j}=0$ ). The DM-1 and DM-2 models could therefore be combined into a DM model, in the following fashion:

$$
\hat{\mu}_{i j}=q \mu_{i i}+(1-q) \mu_{j j}+\left(r_{i}+s_{j}\right)\left(\mu_{i i}-\mu_{j j}\right),
$$

This model contains $r_{i}$ parameters for the origin specific influence and $s_{j}$ parameters for the destination specific influence of cross pressures, thus circumventing the undesirable asymmetry of the DM-1/DM-2 models. The $r_{i}\left(\mu_{i i}-\mu_{j j}\right)$ and $s_{j}\left(\mu_{i i}-\mu_{j j}\right)$ terms are treated as mobility effects, using the simple diagonal reference model as a baseline.

By treating them as simple diagonal reference baseline models plus a non-additive, "cross-pressures" status inconsistency term, the DM-1/DM-2 models can be re-interpreted in a meaningful way within the linear modelling scheme, for which the distinction additive/non-additive is crucial. However, the diagonal reference approach combines multiplicative and additive effects, and this raises the question whether the additive/non-additive distinction is appropriate for this approach, or whether alternatives might be devised that are better suited. This will be discussed in the following paragraph, on a recent alternative model by David Weakliem. 


\subsection{David Weakliem's model}

David Weakliem (1992) formulated an alternative to the DM-1/DM-2 models, that allows salience parameters to vary per category of the common scale. In his model, $\hat{\mu}_{i j}$ is the weighted sum of $\mu_{i i}$ and $\mu_{i j}$. However, the weights $w_{i j}$ are dependent on both origin and destination, and are a function of an overall salience parameter $\phi$ and salience deviation parameters $\pi_{i}$. Weakliem's model is as follows:

$$
\hat{\mu}_{i j}=w_{i j} \mu_{i i}+\left(1-w_{i j}\right) \mu_{j j}
$$

where

$$
w_{i j}=\frac{\phi \pi_{i}}{\phi \pi_{i}+\pi_{j}} \text { and } \prod_{i=1}^{r} \pi_{i}=1
$$

A restriction must be placed on the $\pi_{i}$ parameters. Weakliem let the sum over the parameters equal $r$, but we prefer to let the product over $\pi_{i}$ equal 1 . If all $\pi_{i}$ parameters are equal to 1 , then Weakliem's model is equivalent to the simple diagonal reference model, with $w_{i j}=\phi /(\phi+1)=p$. Values of $\pi_{i}$ less than 1 mean that for category $i$ of the common scale, $X_{1}$ has less than average salience compared to $X_{2}$.

A criticism of the DM-1/DM-2 models was that they are asymmetrical, since they require that differences in salience depend on either origin category or destination category. Weakliem's model can be seen as a synthesis of the DM-1/DM-2 models. The DM-1 model can be rewritten as:

$$
\hat{\mu}_{i j}=w_{i j} \mu_{i i}+\left(1-w_{i j}\right) \mu_{j j}
$$

where

$$
w_{i j}=p_{i}=p \delta_{i}=\frac{\phi \delta_{i}}{\phi \delta_{i}+\delta_{j}}
$$

with

$$
\prod_{i=1}^{r} \delta_{i}=1 \text { and } p=\frac{\phi}{\phi+1}
$$

In this specification, the DM-1 model resembles Weakliem's model, with an overall salience parameter $p$ and salience deviation parameters $\delta_{i}$, which depend only on the $X_{1}$ category. Likewise, the DM-2 model could be respecified as: 


$$
\hat{\mu}_{i j}=w_{i j} \mu_{i i}+\left(1-w_{i j}\right) \mu_{j j}
$$

where

$$
w_{i j}=p_{j}=p \delta_{j}=\frac{\phi \delta_{i}}{\phi \delta_{i}+\delta_{j}} .
$$

Weakliem's model synthesizes these two models, by specifying that $w_{i j}$ is dependent on salience deviation parameters $\pi_{i}$ and $\pi_{j}$ for both $X_{1}$ and $X_{2}$.

Like the DM-1 and DM-2 models, Weakliem 's model has $2 r$ non-redundant parameters, 1 more than the square additive model. It also contains nonadditive effects. This can be seen by re-writing it as:

$$
\hat{\mu}_{i j}=w_{i .} \mu_{i i}+w_{. j} \mu_{j j}+\left(w_{i j}-w_{i}\right) \mu_{i i}+\left(1-w_{i j}-w_{. j}\right) \mu_{j i}
$$

where

$$
w_{i .}=\frac{1}{r} \sum_{j=1}^{r} w_{i j}
$$

and

$$
w_{. j}=\frac{1}{r} \sum_{i=1}^{r} w_{i j}
$$

The terms $w_{i} \mu_{i i}$ and $w_{j .} \mu_{j j}$ are of course additive, but the remainder of equation (16) is not. However, it is not possible to write $w_{i}$ or $w_{. j}$ as simpler expressions of $\pi_{i}$ and $\phi$, and we are unable to specify the non-additive terms in a substantively meaningful way.

Instead, Weakliem's model could be seen as a submodel in a "generalized diagonal reference" approach, that should be seen as an alternative to linear ANOVA modelling. By relaxing the restriction on the $w_{i j}$ parameters are functions of the $\phi$ and $\pi_{i}$ parameters, the diagonal reference approach can be expanded to an equivalent of a saturated ANOVA model. One way of doing this might be:

$$
\hat{\mu}_{i j}=w_{i j} \mu_{i i}+\left(1-w_{i j}\right) \mu_{j j}
$$

where

$$
w_{i j}=p \kappa_{i j}, \quad \kappa_{i i}=1,
$$

and

$$
\prod_{i=1}^{r} \prod_{i=1}^{r} \kappa_{i j}=1 .
$$

In equation (17), $p$ is an overall salience parameter, and $\kappa_{i j}$ are weights for 
the individual cells. There are $r$ parameters $\mu_{i i}, 1$ parameter $p$, and $r^{2}-r$ parameters $\kappa_{i j}$, which means there are as many unknow parameters as known cell values, so that the model will fit $\mu_{i j}$ perfectly. ${ }^{6} W$ eakliem's model is a restricted version of the model in equation (17), that takes into account the overall status effect of the status variables (the $\mu_{i i}$ pa-zmeters) and the differences in salience (the $\phi$ and $\pi_{i}$ parameters). In this mudelling strategy, Weakliem's model does not utilize information from speciic combinations of cells, but only exhausts the explanatory power of the status variables themselves. It could therefore be seen as the counterpart to the square additive baseline model in such a generalized diagonal reierence approach.

We can conclude that Weakliem's model successfully estends the basic idea of the simple diagonal reference model to allow for unequal salience per category of the common scale. The simple diagonal reierence model can be seen as a restricted submodel of Weakliem's model (all salience deviation parameters are equal to 1). The DM-1/DM-2 models can in seen as alternative baseline models, to test the hypothesis that salience deriation parameters vary for origin category only (DM-1), or for destination category only (DM2).

\section{Conclusions}

In this paper, we examined the relationships between the square additive model, which is used as a baseline for detecting status inconsistency and mobility effects, and alternative approaches by Hope and Sobel. These alternatives have in common the use of a construct of overall status as the new baseline criterion. This is a logical stance, since the existence of an underlying overall status dimension would preclude the existence of status inconsistency and therefore of status inconsistency effects.

The effects of an overall status dimension are given by the halfway parameters in Hope's halfway model or by the diagonal parameters $\mu_{i i}$ in Sobel's diagonal reference model. These parameters indicate to what extent the main effects of the square additive model have equal strength. i.e. the equality in the main effects. These parameters can be supplemented by parameters that relate to the inequality of the main effects of the status variables. This is measured by the difference parameters in Hope's approach as a subtraction, and by salience parameters based on a ratio in Sobel's approach. Inequality of the main effects means that one of the status variables has a stronger effect on the dependent variable, has greater salience for the respondent. We agree with Sobel that these effects do not constitute status inconsistency 
effects; they are in any case distinct from status inconsistency effects that are based on specific combinations of cells.

The DM-1/DM-2/Weakliem baseline models each - have one parameter more than a square additive model would have and contain some nonadditive effects. A formal objection to the use of these models in conjunction with non-additive status inconsistency terms is that a model with all interaction terms cannot be fitted. This problem could be solved by using restricted version of a generalized diagonal reference model. Alternatively, the DM-1/DM-2 models could be regarded as simple diagonal reference models plus a non-additive "cross-pressures" term, in addition to other non-additive terms for status inconsistency effects.

Finally, the question to be answered is whether the alternative baseline models provide justification for new status inconsistency and mobility research. The halfway/difference baseline model is equivalent to the square additive model, and a restricted version with a linear constraint on the halfway and/or difference effects cannot be justified unless it does not explain significantly less variance than the square additive model. The simple diagonal reference model usually explains almost as much variance as the square additive model, and when it does not, this will be because salience is not equal for all categories, and either the DM-1, DM-2, or Weakliem's model should be used instead. These models do not explain all the variance of the square additive model, but on the other hand they are less parsimonious, so that it is possible, but highly unlikely, that interactions will be found using these models as a baseline where none were found using the square additive model.

One conclusion is that neither Hope's or Sobel's approach will result in the detection of more interaction effects than the square additive model. However, recent work comparing the relative influence of partners or class origin versus class destination show that these models for the analysis of salience have proven useful in their own right. Sobel's model has the advantage that one is able to model the salience of both status dimensions with one single parameter. Furthermore, Sobel's model is a good example of how to translate theoretical ideas into a mathematical model.

Notes

${ }^{1}$ De Graaf and Ganzeboom (1990) suggested the name "diagonal reference model", instead of Sobel's term "diagonal mobility model" in order to avoid confusion with Goodman's loglinear diagonal mobility models for loglinear analysis of mobility frequency tables.

${ }^{2}$ Ben Pelzer of our computer support department found that NLR by SPSSX is very suitable for estimating Sobel's diagonal reference model. Program speed is high, derivatives do not have 
to be specified, and the accuracy of initial values only marginally affects program speed. For estimating halfway/difference models, we used GLIMMAT, a set of SAS macros using PROC IML to construct design matrices and estimate generalized linear models (Hendrickx 1992a).

All models discussed in the text assume a continuous dependent variable with a normal distribution. Hope's halfway/difference model is also used in loglinear models of mobility frequency tables (Hope 1981, 1982, 1991; Sobel, Hout and Duncan 1985). The substantive issues are different, but the design techniques are identical.

Sobel's nonlinear diagonal reference model can also be used with a dichotomous dependent variable in a logitistic model (De Graaf and Heath 1992; De Graaf, Nieuwbeerta and Heath 1992, Weakliem 1992). Hendrickx (1992b) shows how PROC NLIN by SAS can be used to emulate the GLIM 3.77 program and thus to estimate nonlinear designs in loglinear, logit, or other GLIM-type models. CNLR by SPSSX can also be used to estimate logistic diagonal reference models (De Graaf and Heath 1992; De Graaf, Nieuwbeerta and Heath 1992).

The basic idea of the diagonal reference model, i.e. fitting main effects that are proportional to each other, has also been implemented for asymmetric interaction in loglinear model, in the "proportional asymmetry model" (Hendrickx and Lammers 1992).

3 Jackson and Burke (1965) cite two articles criticizing the inadequate control on effects of the status variables. Robert Edward Mitchel (1964), 'Methodological notes on a theory of status crystallization', Public Opinion Quarterly 28: 315-325 and N. J. Demerath III (1962), 'Status discrepancy and vertical status: criticisms and suggested remedies', paper read at the American Sociological Association meetings, Washington D.C. Other critics were Blalock $(1966,1967)$ and Duncan (1966).

${ }^{4}$ Other constraints, or contrasts (Bock 1975), are also possible. The contrast used will not affect the detection of non-additive effects, but would affect the interpretation of interaction parameters.

5 An alternative way of looking at the diagonal reference model is in terms of the effects of an underlying overall status variable based on weights $p$ and $1-p$, which are determined on empirical grounds. Seen this way, Sobel's simple diagonal reference model would be a counterpart to Hope's halfway model.

6 The parameters from this model can be calculated directly using the observed means. We were unable to derive the parameter values using NLR by SPSSX, presumably because the program assumes an over-identified model. This did succeed if the restriction $\kappa_{12}=1$ was used, rather than restrict the product over all $\kappa_{i j}$ parameters to be 1 . The program showed no perfect correlations between parameters, so the model is indeed identified.

\section{References}

Alschuler, L. R. (1973). Status equilibration, reference groups, and social fields. General Systems 18: 99-118.

Blalock, H. M. (1966). The identification problem and theory building: the case of status inconsistency. American Sociological Review 31: 52-61.

Blalock, H. M. (1967). Status inconsistency and interaction: some alternative models. American Journal of Sociology 73: 305-315.

Blau, P. M. \& O. T. Duncan (1967). The American Occupational Structure. New York: The Free Press.

Bock, R. D. (1975). Multivariate Statistical Methods in Behavioral Research. New York: McGraw-Hill.

De Graaf, N. D. (1991). Distinction by consumption in Czechoslovakia, Hungary, and the Netherlands. European Sociological Review 7: 267-290.

De Graaf, N. D. \& A. Heath (1992). Husbands and wives' voting behaviour in Britain: Class dependent mutual influence of spouses. Acta Sociologica 35: 311-322. 
De Graaf, N. D. \& H. Ganzeboom (1990). Statusgroepen en cultuurdeeiname. (Status group effects on culture consumption.) Mens en Maatschappij 65: 219-244.

De Graaf, N. D., P. Nieuwbeerta \& A. Heath (1992). Intergenerational class mobility and political preferences in Germany, the Netherlands, Britain and the United States. Paper to be presented at the ASA meeting in Pittsburgh, 19-24 August 1992.

De Graaf, N. D. \& W. Ultee (1987). Intergenerationele mobiliteit en politieke verhoudingen. Acta Politica 22: 1-37.

De Graaf, N. D. \& W. C. Ultee (1990). Individual preferences, social mobility and electoral outcomes. Electoral Studies 9: 108-131.

Duncan, O. D. (1966). Methodological issues in the analysis of social mobility. In N. L. Smelser \& S. M. Lipset (eds.), Social Structure and Mobility in Economic Development, Chicago, Aldine Publishing Company, pp. 51-97.

Geschwender, J. A. (1967). Continuities in theories of status inconsistency and cognitive dissonance. Social Forces 46: 160-171.

Hendrickx, J. (1992a). Using SAS macros and PROC IML to create special designs for Generalized Linear Models. In: SAS Institute, SEUGI ' 92 . Proceedings of the SAS ${ }^{\circledR}$ European Users Group International Conference, Vienna, May 19-22, 1992, pp. 634-655.

Hendrickx, J. (1992b). Specifying link functions and error distributions for categorical models using nonlinear designs. Available on request.

Hendrickx, J. \& J. Lammers (1992). Design techniques for equal/unequal main effects and symmetrical/asymmetrical interactions. In: B. Francis, G. U. H. Seeber, P. G. M. van der Heijden \& W. Jansen (eds.), Statistical Modelling - 6th International Workshop on Statistical Modelling, July 15-19, 1991, Utrecht, The Netherlands, Amsterdam, Elsevier, pp. 149-158.

Hope, K. (1971). Social mobility and fertility. American Sociological Review 36: 1019-1032.

Hope, K. (1975). Models for status inconsistency and social mobility effects. American Sociological Review 40: 322-343.

Hope, K. (1981). Vertical mobility in Britain: a structured analysis. Sociology 15: 19-55.

Hope, K. (1982). Vertical and nonvertical class mobility in three countries. American Sociological Review 47: 99-113.

Hope, K. (1991). Design matrix analysis. Quality and Quantity 25: 333-344.

House, J. S. (1978). Facets and flaws of Hope's diamond model. American Sociological Review 43: 439-442.

Jackson. E. F. (1962). Status consistency and symptoms of stress. American Sociological Review 27: 469-480.

Jackson, E. F. \& P. J. Burke (1965). Status and symptoms of stress: additive and interaction effects. American Sociological Review 30: 556-564.

Jackson, E. F. \& R. F. Curtis (1972). Effects of vertical mobility and status inconsistency: a body of negative evidence. American Sociological Review 37: 701-713.

Lenski, G. E. (1954). Status crystallization: a non-vertical dimension of social status. American Sociological Review 19: 405-413.

Lenski, G. E. (1956). Social participation and status crystallization. American Sociological Review 21: 458-464.

Slomczynski, K. M. (1989). Effects of status-inconsistency on the intellective process: The United States, Japan, and Poland. In M. L. Kohn (ed.), Cross-National Research in Sociology, London: Sage Publications, pp. 148-166.

Sobel, M. E. (1981). Diagonal mobility models: a substantively motivated class of designs for the analysis of mobility effects. American Sociological Review 46: 893-906.

Sobel, M. E. (1985). Social mobility and fertility revisited: some new models for the analysis the mobility effects hypothesis. American Sociological Review 50: 699-712.

Sobel, M. E., M. Hout \& O. D. Duncan (1985). Exchange, structure, and symmetry in occupational mobility. American Journal of Sociology 91: 359-372.

Sorenson, A. M. (1989). Husbands' and wives' characteristics and fertility decisions: a diagonal mobility model. Demography 26: 125-135. 
Weakliem, D. (1992). Does social mobility affect political behaviór? European Sociological Review 8: 153-165.

Whitt, H. P. (1983). Status inconsistency: a body of negative evidence or a statistical artifact? Social Forces 62: 201-233.

Wilson, K. L. (1979). Status inconsistency and the Hope technique I: the grounds for a resurrection. Social Forces 57: 1229-1247.

Wilson, K. L. \& L. A. Zurcher (1976). Status inconsistency and participation in social movements: an application of Goodman's hierarchical modeling. The Sociological Quarterly 17: 520-533.

Zimmerman, E. (1985). Almost everything you always wanted to know about status inconsistency (but were afraid to measure). Social Behavior and Personality 13: 193-214.

Zurcher, L. A. \& K. L. Wilson (1979). Status inconsistency and the Hope technique II: a linear hypothesis about status enhancement, status detraction, and satisfaction with membership. Social Forces 57: 1248-1664. 